\title{
An exact renormalisation group for Bloch electrons in a magnetic field
}

\author{
Michael Wilkinson \\ Department of Physics and Applied Physics, John Anderson Building, University of \\ Strathclyde, Glasgow, G4 0NG, UK
}

Received 15 January 1987, in final form 19 March 1987

\begin{abstract}
Bloch electrons in a magnetic field can be modelled by a one-dimensional Hamiltonian $\hat{H}=H(\hat{x}, \hat{p})$, which is periodic in $\hat{x}$ and $\hat{p}$. When $\beta$, the ratio of Planck's constant $h$ to the area of a unit cell is a rational number $p / q$, the spectrum is a set of $q$ Bloch bands. If $\beta$ is perturbed away from this rational value, the spectrum becomes a Cantor set, but it is still possible to define a set of generalised Bloch waves which form a complete basis set for a given band. Projecting these states into the band, and taking matrix elements of the Hamiltonian leads to a new effective Hamiltonian $H_{\nu}^{\prime}(\hat{x}, \hat{p})$ and Planck constant $h_{\nu}^{\prime}$ describing the spectrum of the $\nu$ th band. Since degrees of freedom have been eliminated by projecting into a band, this is a renormalisation group (RG) transformation. The RG transformation can be iterated indefinitely if the measure of the spectrum is zero or if $\beta$ is a Liouville number. The measure of the spectrum vanishes if $H(x, p)$ has centres of threefold or fourfold symmetry in the phase plane, and the RG transformation explains the hierarchical structure of the spectrum observed in these cases.
\end{abstract}

\section{Introduction}

This paper considers the spectrum of a Hamiltonian operator $\hat{H}=H(\hat{x}, \hat{p})$ which is periodic in both $\hat{x}$ and $\hat{p}$; an example is

$$
\hat{H}=2(\cos \hat{p}+\alpha \cos \hat{x}) \text {. }
$$

Hamiltonians of this type arise as single band effective Hamiltonians for the problem of Bloch electrons in a magnetic field in two dimensions. If the cyclotron energy is large compared to the periodic potential, the splitting of the degeneracy of the Landau levels by the potential is described by an effective Hamiltonian of this type (Rauh $1974,1975)$, and $H(\hat{x}, \hat{p})$ has the same rotational symmetry in phase space as the potential $V(x, y)$ has in coordinate space (Wilkinson 1987). The ratio $\beta$ of the effective Planck constant to the area of a unit cell in phase space is the same as that of the area of a flux quantum to the unit cell in coordinate space. Effective Hamiltonians of the same type also arise when the magnetic field is a perturbation on the band spectrum due to the potential (Peierls 1933), but in this case $\beta$ is equal to the inverse ratio of the area of the flux quantum to that of a unit cell (Hofstadter 1976).

The Schrödinger equation corresponding to this type of Hamiltonian can be written as a difference equation with periodic coefficients; for instance in the $x$ representation equation (1.1) gives

$$
\psi(x+\hbar)+\psi(x-\hbar)+2 \alpha \cos x \psi(x)=E \psi(x)
$$


where $\hbar=2 \pi \beta$, or equivalently

$$
\psi_{n+1}+\psi_{n-1}+2 \alpha \cos (2 \pi \beta n+\Delta) \psi_{n}=E \psi_{n}
$$

which is called Harper's equation (Harper 1955). When $\beta$ is a rational number $p / q$, Bloch's theorem applies to equation (1.3), and the spectrum consists of a set of $q$ bands with dispersion relation $E=\varepsilon_{\nu}(k, \Delta)$, where $k$ is the Bloch wavevector and $\nu$ an index labelling the band. When $\beta$ is irrational, the spectrum is a Cantor set (Simon 1982). Numerical calculations by Hofstadter (1976) suggest that when $\alpha=1$ this spectrum has a hierarchical structure, which corresponds to the critical point of a renormalisation group ( $R G$ ) transformation. This paper describes the construction of an exact renormalisation group transformation suitable for analysing the spectrum of Bloch electrons in a magnetic field. It extends earlier work, in which the RG transformation was described for a special case (Wilkinson 1986). Several other authors, notably Suslov (1982), and Ostlund and Pandit (1984), have made contributions to the analysis of Harper's equation using renormalisation group methods different from those introduced in this paper. The advantages of the method described here will be discussed in the conclusion $(\$ 7)$.

The first step in obtaining this RG transformation is to consider the effect of perturbing $\beta$ away from a rational value: write

$$
\beta=p / q+\Delta \beta
$$

where $\Delta \beta$ is a small parameter. For each band it is possible to define a set of generalised Bloch waves, which locally resemble the Bloch states of the unperturbed, rational system, but on a larger scale have a slowly varying phase parameter $\Delta$. These generalised Bloch states tend towards the Bloch waves of the rational system as $\Delta \beta \rightarrow 0$, and provided $\Delta \beta$ is sufficiently small they can be shown to form a complete basis for the band. Section 2 will describe the Bloch states of the rational system; this material is included in order to establish notation. The generalised Bloch waves for the case where $\beta$ is irrational are constructed in $\S 3$.

The generalised Bloch waves for the $\nu$ th band can be made orthogonal to all states outside this band by means of a projection operator. The projection operator acts as a small perturbation on these generalised Bloch waves, provided that $\Delta \beta$ is sufficiently small. This is discussed in $\S 4$, which also estimates how large $\Delta \beta$ can be before the projection operator ceases to be a small perturbation. These estimates are important because they help to determine the class of irrational numbers for which the $R G$ transformation can be iterated.

Section 5 contains the equations defining the RG transformation. Taking matrix elements of the Hamiltonian in the basis of the projected generalised Bloch waves leads to a new Schrödinger equation, which gives an exact description of only one band of the spectrum (see figure 1). This Schrödinger equation is in the form of a difference equation with periodic coefficients, and can be expressed as a Hamiltonian periodic in $x$ and $p$ (by reversing the calculation which leads from (1.1) to (1.3)). This Hamiltonian $H_{\nu}^{\prime}$ is related to the Bloch dispersion relation of the band by replacing the parameters $\Delta, k$ by operators $\hat{x}, \hat{p}$

$$
\hat{H}_{\nu}^{\prime}=\varepsilon_{\nu}(\hat{p} / q, \hat{x} / q)+\mathrm{O}(\Delta \beta) .
$$

This is reminiscent of the Peierls substitution method for obtaining an effective Hamiltonian for Bloch electrons in a magnetic field (Peierls 1933). The Planck constant for 




Figure 1. (a) When $\beta$ is a rational number $p / q$, the spectrum consists of $q$ bands. (b) When $\beta$ is perturbed away from this rational value, the spectrum becomes much more complicated, and is a Cantor set if $\beta$ is irrational. The gaps between bands persist under this perturbation. (c) The RG transformation produces a new Hamiltonian which has the same spectrum as one band of the original Hamiltonian.

this renormalised Hamiltonian, defined by $[\hat{x}, \hat{p}]=\mathrm{i} \hbar_{\nu}^{\prime}=2 \pi \mathrm{i} \beta_{\nu}^{\prime}$, is given by

$$
\beta_{\nu}^{\prime}=\frac{q \beta-p}{\left[\left(1-q M_{\nu}\right) / p\right] \beta+M_{\nu}}
$$

where $M_{\nu}$ is the quantised Hall conductance integer of the $\nu$ th band. (The quantised Hall effect in a periodic potential has been analysed by Thouless et al (1982).) Equations (1.5) and (1.6) are the principal results of this paper.

This procedure maps $H(\hat{x}, \hat{p})$ and $\hbar$ into a new Hamiltonian and Planck constant $H_{\nu}^{\prime}(\hat{x}, \hat{p})$ and $\hbar_{\nu}^{\prime}$, describing the spectrum of the $\nu$ th band. Since this mapping eliminates degrees of freedom (by projecting into a band), it is a renormalisation group (RG) transformation. This RG transformation can be iterated, provided that $\Delta \beta$ is sufficiently small at every stage. This iteration is discussed in $\S 6$. There are two situations where this is possible.

(a) Usually, as the RG transformation is iterated, the gaps in the spectrum get smaller, and the results of $\S 4$ show that the smaller the gaps between bands, the smaller $\Delta \beta$, the deviation from rationality, must be. This implies that $\beta$ must usually be a Liouville number.

(b) If, for any reason, the gaps between bands do not occupy a decreasing fraction of the range of the spectrum as the RG transformation is iterated, then the iteration can be continued indefinitely for almost all values of $\beta$. This happens if the Hamiltonian has axes of threefold or fourfold symmmetry in phase space, and the RG transformation explains the hierarchical structures observed in the spectrum in these cases.

Finally, $\S 7$ is a conclusion, and sets the results of this paper in context with earlier work on this problem.

\section{The spectrum when $\beta$ is rational}

In this section, the case of rational $\beta$ will be discussed, mainly in order to introduce notation. It may be helpful to think of Harper's equation (1.3) as a specific example, but the results are applicable to any Hamiltonian periodic in $x$ and $p$. Most of the results described in this section are well known: Hofstadter (1976) gives a useful list of references. 
When $\beta=\beta_{0}=p / q$, where $p$ and $q$ are coprime integers, the cosine term in (1.3) is periodic in $n$ with period $q$, so that Bloch's theorem applies. The spectrum consists of $q$ bands, labelled by an integer $\nu=1, \ldots, q$. The eigenfunctions are Bloch waves

$$
\psi_{n}=\psi_{n}^{(\nu)}(k, \Delta)=\mathrm{e}^{i k n} U_{n}^{(\nu)}(k, \Delta)
$$

where the $U_{n}$ are periodic with period $q$

$$
U_{n+q}^{(\nu)}(k, \Delta)=U_{n}^{(\nu)}(k, \Delta)
$$

and the Bloch wavevector $k$ and phase parameter $\Delta$ vary over the following ranges:

$$
0 \leqslant k<2 \pi / q \quad 0 \leqslant \Delta<2 \pi p / q .
$$

The following properties of these Bloch waves will be important in defining the generalised Bloch waves for irrational values of $\beta$.

(a) As well as being periodic in $\Delta$ with period $2 \pi p / q$

$$
U_{n}^{(\nu)}(k, \Delta+2 \pi p / q)=U_{n}^{(\nu)}(k, \Delta)
$$

The $U_{n}$ also satisfy (apart from an irrelevant overall phase)

$$
U_{n}^{(\nu)}\left(k, \Delta+\frac{2 \pi}{q}\right)=U_{n+s}^{(\nu)}(k, \Delta) \quad \mathrm{sp}=1 \bmod q .
$$

This implies that there is a $p$-fold degeneracy in the spectrum and that the Bloch dispersion relation $E=\varepsilon_{\nu}(k, \Delta)$ is periodic with period $2 \pi / q$ in $\Delta$

$$
\varepsilon_{\nu}(k, \Delta+2 \pi / q)=\varepsilon_{\nu}(k, \Delta)=\varepsilon_{\nu}(k+2 \pi / q, \Delta) .
$$

(b) When $k=0,2 \pi / q$ or when $k=2 \pi / 2 q$, the $\psi_{n}$ are real (or all have the same complex phase). This is because at these values of $k$ the $\psi_{n}$ can be obtained as an eigenvector of a real symmetric matrix.

(c) Now we consider a collective property of the Bloch waves, for which we shall use the Dirac notation $|k, \Delta ; \nu\rangle$. Provided the bands do not touch, the state $|k, \Delta ; \nu\rangle$ can be chosen to be an analytic function of $k$ and $\Delta$ (this need not be the case, since although $k$ and $\Delta$ fix the ratios of the $\psi_{n}$, every $\psi_{n}$ can be multiplied by the same arbitrary phase factor, $\left.\mathrm{e}^{\mathrm{i} \theta}\right)$. Suppose that $|k, \Delta ; \nu\rangle$ is an analytic function of $k, \Delta$. We can choose the phase so that

$$
|k, \Delta+2 \pi p / q ; \nu\rangle=|k, \Delta ; \nu\rangle
$$

but it is not always possible for $|k, \Delta ; \nu\rangle$ to be periodic in $k$ as well as $\Delta$; in general we have

$$
|k+2 \pi / q, \Delta ; \nu\rangle=\mathrm{e}^{\mathrm{i} M_{\nu} \Delta q / p}|k, \Delta ; \nu\rangle .
$$

The integer $M_{\nu}$ is a topological invariant called the Chern character (Thouless 1983), and is the quantised Hall conductance integer associated with the $\nu$ th band (Thouless et al 1982).

This concludes our discussion of the properties of the Bloch states. In $\S 3$, a set of generalised Bloch states will be constructed, in which the phase parameter $\Delta$ is a slowly varying function of $n$. As a preliminary, the results above will be expressed in a new notation which will prove convenient for defining the generalised Bloch states. Write

$$
\begin{aligned}
& \psi_{n}=\psi_{\nu}\left(k, x_{n}\right) \\
& x_{n}=2 \pi \beta n+\Delta
\end{aligned}
$$


where $\psi_{\nu}(k, x)$ is an analytic function of $k$ and $x$. Consider the construction of the function $\psi_{\nu}(k, x)$. Fix $k$, and vary the phase parameter $\Delta$ : we have

$$
\psi_{\nu}\left(k, x_{n}(\Delta)\right)=\psi_{n}^{(\nu)}(k, \Delta) Z(\Delta)
$$

where $Z(\Delta)$ is a complex number of modulus unity. We can increase $\Delta$ from 0 without any difficulty until $\Delta=2 \pi \beta$, where we have to choose $Z(\Delta)$ in such a way that $\psi_{\nu}(k, x)$ is an analytic function of $x$, without any phase discontinuity. Similarly, we can construct $\psi_{\nu}$ as an analytic function of $k$. The $\psi_{\nu}(k, x)$ is not unique, because of the arbitrary overall phase of the Bloch waves.

The properties of the Bloch waves described above all imply corresponding properties of the function $\psi_{\nu}(k, x)$. The Bloch condition is

$$
\psi_{\nu}(k, x+2 \pi p)=\mathrm{e}^{i k q} \psi_{\nu}(k, x)
$$

and the $p$-fold degeneracy expressed by equation $(2.5)$ is represented by the relation

$$
\psi_{\nu}(k, x+2 \pi)=\mathrm{e}^{\mathrm{i} \theta} \psi_{\nu}(k, x)
$$

where $\theta$ is an arbitrary phase. The requirement that the Bloch waves be real (apart from the overall phase) when $k=0,2 \pi / 2 q, 2 \pi / q$ can be satisfied by writing, at these values of $k$

$$
\psi_{\nu}(k, x)=\mathrm{e}^{\mathrm{i} m x / 2 \beta} A(x)
$$

with $A(x)$ real and periodic or antiperiodic with period $2 \pi$. The integers $m$ in $(2.14)$ depend on the value of $k$ and on the band. Comparing (2.14) with (2.8), we find that the Chern character is related to these integers:

$$
M_{\nu}=\frac{1}{2}[m(k=2 \pi / q)-m(k=0)] .
$$

\section{Generalised Bloch waves}

The object of this section is to define a set of states which have the following properties.

(a) They correspond to the Bloch states discussed in $\S 2$ when $\beta$ is rational, and when $\beta$ is perturbed away from a rational value these states tend toward the Bloch states as $\Delta \beta \rightarrow 0$.

(b) They are periodic in $k$ and $\Delta$ (apart from a phase discontinuity if the Chern character is non-zero), and because of $(a)$ they must have the same Chern character as the Bloch waves of the unperturbed band.

(c) They span all of the states in the $\nu$ th band of the spectrum.

(d) They are orthogonal to all states outside the $\nu$ th band.

These states will be termed generalised Bloch waves. Requirements $(c)$ and $(d)$ imply that the generalised Bloch waves can be used as a complete basis set for the $\nu$ th band of the spectrum, which is assumed to be separated from other bands by a pair of gaps.

Using the notation introduced in $\S 2$, we will write the amplitudes $\psi_{n}$ defining the generalised Bloch wave in the form

$$
\psi_{n}=\psi_{\nu}^{\prime}\left(k, x_{n}\right)
$$


where $\psi_{\nu}^{\prime}(k, x)$ is an analytic function of $k$ and $x$, closely related to the function $\psi_{\nu}(k, x)$ introduced in (2.9), and is in the form of a Bloch wave

$$
\begin{aligned}
& \psi_{\nu}^{\prime}(k, x)=\mathrm{e}^{\mathrm{i} k x / 2 \pi \beta} U_{\nu}^{\prime}(k, x) \\
& U_{\nu}^{\prime}(k, x+2 \pi)=U_{\nu}^{\prime}(k, x)
\end{aligned}
$$

and

$$
\begin{aligned}
& x_{n}=2 \pi \beta n+\Delta \\
& \beta=p / q+\Delta \beta .
\end{aligned}
$$

Thus the Bloch function $\psi_{\nu}^{\prime}(k, x)$ is sampled irrationally, so that the $\psi_{n}$ are a quasiperiodic sequence (figure 2). Taking $\psi_{\nu}^{\prime}(k, x)=\psi_{\nu}(k, x)$ is not correct, for the following reason. Requirement $(b)$ states that the generalised Bloch states are to be periodic in $k$ and $\Delta$ (apart from an overall phase). If the values of the integers $m$ introduced in (2.14) are different for the $k=0$ and $k=2 \pi / q$ band edges, this requirement will not be satisfied unless $\psi_{\nu}(k, x)$ is modified. If we take, at the edges of the band,

$$
\psi_{\nu}^{\prime}(k, x)=\mathrm{e}^{\mathrm{i} m x / 2 \beta} A(x)
$$

where $A(x)$ is the real valued function introduced in (2.14), then the $\psi_{n}$ remain real at the band edges, apart from an overall phase, and the states at opposite edges of the band correspond. This has the effect of changing the Bloch wavevector at the edges of the band: we have

$$
\begin{aligned}
\psi_{\nu}^{\prime}(k, x+2 \pi p) & =\mathrm{e}^{2 \pi \mathrm{i} p m / 2 \beta} \psi_{\nu}(k, x) \\
& =\mathrm{e}^{\mathrm{i} \Delta k p / \beta} \psi_{\nu}^{\prime}(k, x)
\end{aligned}
$$

so that the Bloch wavevector of states at the edge of the band has been shifted by an
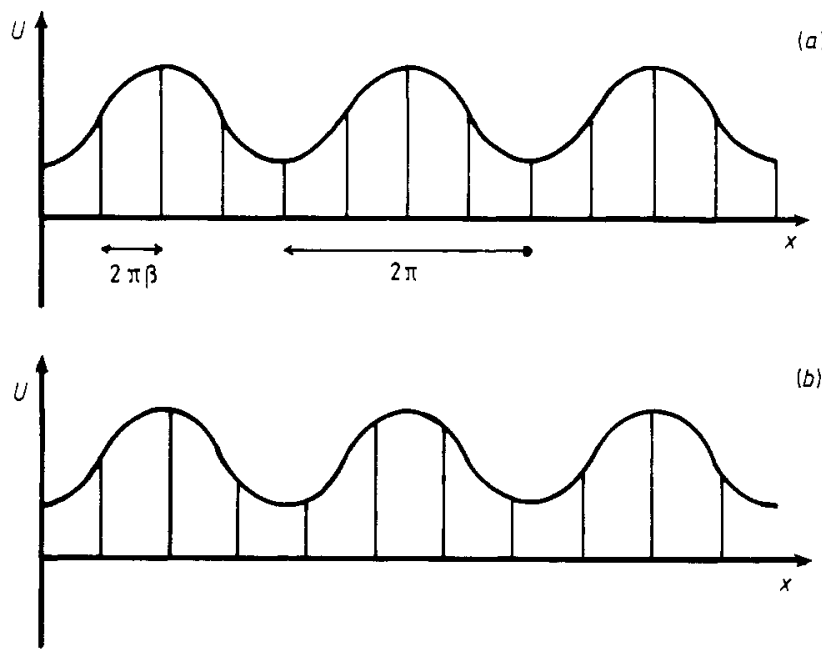

Figure 2. (a) When $\beta$ is rational, the amplitudes of the Bloch states are of the form $\psi_{n}=\mathrm{e}^{i k n} U_{n}$, and the periodic sequence $\left\{U_{n}\right\}$ can be obtained by sampling a periodic function. (b) The sequence $\left\{U_{n}\right\}$ for the generalised Bloch waves is obtained by sampling the same periodic function irrationally, giving a quasiperiodic sequence. 
amount

$$
\Delta k=2 \pi \frac{m}{2}\left(1-\frac{\beta q}{p}\right)
$$

which tends to zero as $\Delta \beta \rightarrow 0$.

Equations (3.4)-(3.6) specify $\psi_{\nu}^{\prime}(k, x)$ at the edges of the band, and show that if $m \neq 0$, the Bloch wavevector at the band edges must be shifted. Now $\psi_{\nu}^{\prime}(k, x)$ will be specified in terms of $\psi_{\nu}(k, x)$ for all values of $k$. Since the range of values of $k$, the Bloch wavevector, is different it is necessary to set up a correspondence between the new $k^{\prime}$ and old values $k$. The new value of the Bloch wavevector $k^{\prime}$ must correspond to the old value of $k$ in the limit $\Delta \beta \rightarrow 0$ : the simplest choice is

$$
k^{\prime}=\Delta k_{0}+\frac{q}{2 \pi}\left(\frac{2 \pi \beta}{p}+\left(\Delta k_{1}-\Delta k_{0}\right)\right) k
$$

where $\Delta k_{0}$ is the value of $\Delta k$ at the $k=0$ edge of the band, and $\Delta k_{1}$ is the value of $\Delta k$ at the $k=2 \pi / q$ edge. Now we can write our final expression for $\psi_{\nu}^{\prime}(k, x)$

$$
\psi_{\nu}^{\prime}\left(k^{\prime}, x\right)=\mathrm{e}^{\left[i\left(k^{\prime}-k\right) x\right] / 2 \pi \beta} \psi_{\nu}(k, x) .
$$

If the Chern character $M_{\nu}$ is not equal to zero, the number of states per unit area in the band changes, since this is proportional to the range of Bloch wavevector $k$. The ratio of the number of states in the $\nu$ th band to the total number of states when $\Delta \beta=0$ is

$$
\mu_{\nu^{\prime}}=\frac{\beta}{p}+M_{\nu}\left(1-\frac{\beta q}{p}\right)
$$

This result should be compared with the Strěda formula (Strěda 1982), which relates the number of states $N_{\nu}$ per unit area to the quantised Hall conductance integer, $M_{\nu}$ :

$$
\sigma_{\nu}=\frac{e^{2}}{h} M_{\nu}=e \frac{\partial N_{\nu}}{\partial B} .
$$

( $B$ is the magnetic field, and $e$ the electronic charge.) For a Landau level, the total density of states is $e B / h$, and the parameter $\beta$ is inversely proportional to $B$ (Wilkinson 1987), so that (3.11) can be written

$$
\begin{aligned}
M_{\nu} & =\frac{h}{e} \frac{\partial N_{\nu}}{\partial B}=\frac{B}{N} \frac{\partial N_{\nu}}{\partial B}=\frac{B}{N} \frac{\partial\left(N \mu_{\nu}\right)}{\partial B} \\
& =\mu_{\nu}+B \frac{\partial \mu_{\nu}}{\partial B}=\mu_{\nu}-\beta \frac{\partial \mu_{\nu}}{\partial \beta} .
\end{aligned}
$$

This shows that (3.10) is in agreement with the Strěda formula (3.11), and that provided they are linearly independent there is exactly the correct number of generalised Bloch states to form a complete basis for the $\nu$ th band.

To summarise: this section has shown how to construct a set of generalised Bloch states by sampling the Bloch function $\psi_{\nu}^{\prime}(k, x)$ irrationally (equations (3.1)-(3.4)). If $M_{\nu}$ is non-zero $\psi_{\nu}(k, x)$ must be modified if the generalised Bloch states are to be periodic (apart from a phase) in $k$ and $\Delta$ (equations (3.5)-(3.9)). This modification alters the number of states in the band, in agreement with the Strěda formula (equations $(3.10)-(3.12))$. 
In order to complete the construction of the generalised Bloch states, it is necessary to apply a projection operator to project into the $\nu$ th band (to satisfy requirement $(d)$ above), and then to show that the states are linearly independent (and therefore satisfy requirement $(c)$ ). Both of these steps require further calculations, and they will be discussed in $\$ \S 4$ and 5 respectively.

\section{Projection into a band}

The generalised Bloch states can be made orthogonal to all states outside the $\nu$ th band by applying a projection operator, $\hat{P}$. It will be shown that provided $\Delta \beta$ is sufficiently small, the projection operator acts as a small perturbation, in the sense that the amplitudes $\psi_{n}^{\prime}$ of the projected states are close to those of the unprojected states $\psi_{n}$.

The projection operator for the $\nu$ th band can be written as a function of the Hamiltonian

$$
\hat{P}_{\nu}=f_{\nu}(\hat{H})
$$

where $f_{\nu}(E)$ is unity if $E$ is inside the $\nu$ th band, zero throughout the rest of the spectrum, and undefined elsewhere. Because the $\nu$ th band is assumed to be separated from the rest of the spectrum by a pair of gaps, $f(E)$ can be a smooth function with an arbitrarily high number of continuous derivatives (figure 3 ). Since $\hat{P}_{\nu}$ is a function of $\hat{H}$, it too is a difference operator with periodic coefficients.



Figure 3. Because the spectrum has gaps $(a)$, the projection operator for a band can be defined using a smooth function $f(E)(b)$. This implies that the projection operator is localised, so that the projection into a band is well defined.

When $\Delta \beta=0$, the projected states $\hat{P}_{\nu}|k, \Delta ; \nu\rangle$ are the same as the unprojected states, since these are all eigenfunctions in the $\nu$ th band. When $\Delta \beta$ is sufficiently small, the projected states are close to the unprojected ones. The reason for this is that the projection operator is localised over a range $l_{p}$ in $x$. For small $\Delta \beta$, the generalised Bloch waves resemble the rational ones on short length scales, less than some coherence length $l_{c}$. Provided $l_{p} \ll l_{c}$, the difference between the generalised Bloch state and the original rational one is small, and the action of the projection operator alters the state by a small amount.

To demonstrate this, it is first necessary to show that $\hat{P}$ is localised, and to estimate the localisation length, $l_{p}$. This will be compared with the coherence length, $l_{c}$, and the limiting value of $\Delta \beta$ estimated. Both $l_{p}$ and $l_{c}$ depend on the form of the dispersion relation $\varepsilon_{\nu}(k, \Delta)$. 


\subsection{Localisation length}

The projection operator can be written

$$
\begin{aligned}
\hat{P}=f(\hat{H}) & =\int \mathrm{d} t \tilde{f}(t) \mathrm{e}^{-\mathrm{i} \hat{H} t} \\
& =\int \mathrm{d} t \tilde{f}(t) \hat{U}(t)
\end{aligned}
$$

where $\tilde{f}(t)$ is the Fourier transform of $f(E)$ and $\hat{U}(t)$ is the evolution operator. Because of the gaps in the spectrum, $f(E)$ can be $C^{\infty}$ so that $\tilde{f}(t)$ decays faster than any power of $|t|$ as $|t| \rightarrow \infty$. For finite times, the evolution operator $\hat{U}(t)$ is localised over a length scale bounded above by

$$
l(t) \leqslant \beta t .
$$

(If the eigenstates are localised, $l(t)$ tends toward the localisation length as $t \rightarrow \infty$, but if the spectrum is continuous, $l(t)$ continues to increase as $t$.) The function $f(t)$ decays faster than any power of $|t|$ for times greater than $t^{*}=1 / \Delta E$, where $\Delta E$ is the smaller of the two gaps flanking the band. From (4.2) and (4.3), it follows that $\hat{P}_{\nu}$ is localised, decaying faster than any power, with a localisation length

$$
l_{p} \sim l\left(t^{*}\right) \leqslant \beta \Delta E .
$$

There are two limiting cases which should be considered (see figure 4). Firstly, if the gaps in the spectrum are large compared to the width of the bands, then $\hat{P}_{1}$, is

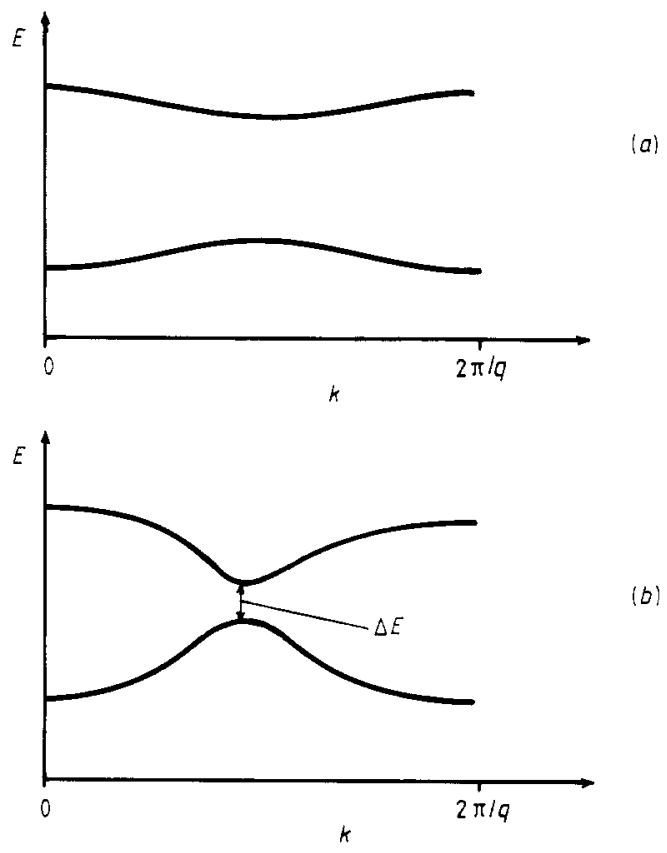

Figure 4. The RO equations are only shown to be valid if $\Delta \beta$ is sufficiently small. If the separation of the bands is large $(a), \Delta \beta$ should satisfy the condition $q^{2} \Delta \beta \ll 1$. If the bands are separated by a narrow gap of size $\Delta E(b)$, then $\Delta \beta$ must satisfy the more stringent condition $q \Delta \beta / \Delta E \ll 1$. 
localised over one unit cell:

$$
\Delta E=\mathrm{O}(1 / q) \quad l_{p}=\mathrm{O}(p) .
$$

The other limit is when the gaps are much smaller than the bands; in this case

$$
l_{p}=\mathrm{O}\left(\frac{p}{q} \Delta E^{-1}\right) \text {. }
$$

\subsection{Coherence length}

The coherence length $l_{c}$ is the length scale over which the generalised Bloch states become noticeably different from the rational case. The effective value of the phase $\Delta$ changes slowly with $n$ : the change in this phase is

$$
2 \pi \Delta \beta n=(\Delta \beta / \beta) x_{n} .
$$

The Bloch waves vary as a function of $\Delta$ over a range $\Delta^{*}$, so that $l_{c}$ is given by

$$
l_{c}=\frac{\beta}{\Delta \beta} \Delta^{*} .
$$

Two limiting cases should be considered. First if the dispersion relation $\varepsilon_{\nu}(k, \Delta)$ is a weak function of $\Delta$, then clearly

$$
\Delta^{*}=\mathrm{O}(1 / q)
$$

so that

$$
l_{c}=O\left(\frac{\beta}{q \Delta \beta}\right) .
$$

If $\varepsilon_{\nu}(k, \Delta)$ is strongly dependent on $\Delta$, in the sense that by varying $\Delta$ two bands can be made to nearly touch with a small gap $\Delta E$, then the wavefunction changes very rapidly in the region of this near degeneracy. In this case.

$$
\Delta^{*}=\mathrm{O}(\Delta E)
$$

so that the coherence length is much shorter

$$
l_{c}=\mathrm{O}\left(\frac{\beta \Delta E}{\Delta \beta}\right)
$$

\section{Matrix elements in a basis of generalised Bloch states}

In this section the matrix elements of the Hamiltonian are evaluated in a basis of generalised Bloch waves. Since these states are not an orthogonal set, the normalisation operator must also be calculated.

If the generalised Bloch states are projected into a band, as described in $\S 4$, the Hamiltonian is block diagonal in this representation with respect to the band index $\nu$. Also, states with different values of the phase parameter $\Delta$ mod $\hbar$ are clearly orthogonal, and since the Hamiltonian is a sum of translation operators which shift states through 
integer multiples of $\hbar$, the Hamiltonian is also block diagonal in $\Delta$. With suitable box normalisation (necessary because $\Delta$ is a continuous variable) we can write

$$
\left\langle k, \Delta, \nu|\hat{H}| k^{\prime}, \Delta^{\prime}, \nu^{\prime}\right\rangle=\delta_{\nu \nu^{\prime}} \delta\left(\Delta-\Delta^{\prime}\right) a_{\nu}\left(k, k^{\prime}, \Delta\right)
$$

where $\delta(x)$ is a Dirac $\delta$ function.

Because the generalised Bloch waves are formed by sampling a Bloch function irrationally to give a quasiperiodic sequence of amplitudes (equation (3.1)), the matrix elements are independent of the phase parameter $\Delta$ in (5.1). Also, because the generalised Bloch waves are quasiperiodic, only states with wavevectors $k$ separated by certain increments are coupled. The states have two periodicities in $x$ : one of period $2 \pi \beta$ corresponding to the periodicity in $\Delta$, and one of period $2 \pi$ corresponding to that of the Bloch function $\psi_{\nu}(k, x)$. The Hamiltonian also has the same periodicities. A pair of generalised Bloch waves is therefore only coupled by the Hamiltonian if the difference between their Bloch wavevectors is

$$
\Delta k=k^{\prime}-k=2 \pi(n p+m \beta)
$$

where $n$ and $m$ are integers. The generalised Bloch states are periodic in $k$ with period

$$
K_{\nu}=2 \pi\left[\frac{\beta}{p}+M_{\nu}\left(1-\frac{\beta q}{p}\right)\right] .
$$

Using the gap labelling theorem (Johnson and Moser 1982, Simon 1982), and the Strěda formula (Strěda 1982), it can be shown that $\left(1-q M_{\nu}\right)$ is an integer multiple of p. This implies that $\Delta k$ can be written in the form

$$
\Delta k=n^{\prime} \kappa+m^{\prime} K_{v}
$$

where $n^{\prime}, m^{\prime}$ are integers and

$$
\kappa=2 \pi(q \beta-p) \text {. }
$$

Since the generalised Bloch states are periodic in $k$ with period $K_{\nu},(5.4)$ reduces to

$$
\Delta k=n^{\prime} \kappa \bmod K_{v} .
$$

The matrix elements (5.1) can therefore be written in the form

$$
\left\langle k, \Delta ; \nu|\hat{H}| k^{\prime}, \Delta^{\prime} ; \nu^{\prime}\right\rangle=\delta_{\nu \nu^{\prime}} \delta\left(\Delta-\Delta^{\prime}\right) \delta\left(k-k^{\prime}-n_{\kappa}\right) H_{n, \nu}\left(\frac{k+k^{\prime}}{2}\right)
$$

where $H_{n, \nu}(k)$ is periodic in $k$ with period $K_{\nu}$. The normalisation matrix elements have the same form

$$
\left\langle k, \Delta ; \nu \mid k^{\prime}, \Delta^{\prime} ; \nu^{\prime}\right\rangle=\delta_{\nu \nu} \delta\left(\Delta-\Delta^{\prime}\right) \delta\left(k-k^{\prime}-n \kappa\right) N_{n, \nu}\left(\frac{k+k^{\prime}}{2}\right)
$$

where $N_{n, \nu}(k)$ has the same periodicity as $H_{n, \nu}(k)$. In this basis, the Hamiltonian and normalisation operators are sums of translation operators in $k$, with periodic coefficients. The splitting of each band is therefore described by a new difference equation with periodic coefficients, analogous to (1.2), but with a different ratio of periodicities $\beta_{v}^{\prime}$, given by

$$
\left|\beta_{\nu}^{\prime}\right|=\frac{\kappa}{K_{\nu}} \bmod 1=\frac{q \beta-p}{(\beta / p)\left(1-q M_{\nu}\right)+M_{\nu}} .
$$


This renormalised value of $\beta$ tends to zero as $\beta \rightarrow p / q$. An interesting special case is when $p=1$ and the quantised Hall conductance $M_{\nu}$ is zero, when

$$
\beta_{\nu}^{\prime}=\frac{1}{\beta} \bmod 1 .
$$

In this case the renormalisation of $\beta$ is related to its continued fraction expansion (Wilkinson 1986).

The matrix elements are described by the functions $H_{n, \nu}(k)$ and $N_{n, \nu}(k)$ introduced in (5.7) and (5.8). These functions can be calculated easily in the limit $\Delta \beta \rightarrow 0$. First consider the normalisation operator. In the rational case the Bloch waves are mutually orthogonal, and when $\Delta \beta$ is small, the generalised Bloch waves locally resemble the rational ones. It follows that the generalised Bloch waves are orthogonal in the limit $\Delta \beta \rightarrow 0$ :

$$
N_{n, v}(k)=\delta_{n 0}+\mathrm{O}(\eta)
$$

where the error parameter $\eta$ is the ratio of $\kappa$ to the range $\Delta k^{*}$ over which $\varepsilon_{\nu}(k, \Delta)$ varies as a function of $k$,

$$
\eta=\kappa / \Delta k^{*} \text {. }
$$

When the gaps $\Delta E$ obtained by varying $k$ are small, this range is $\Delta k^{*}=\Delta E$, so that

$$
\eta=\mathrm{O}(q \Delta \beta / \Delta E)
$$

and when the gaps are not small,

$$
\eta=\mathrm{O}\left(q^{2} \Delta \beta\right) .
$$

Now consider the Hamiltonian. Using the fact that the generalised Bloch waves tend toward the rational case as $\Delta \beta \rightarrow 0$, with a slowly varying phase

$$
\Delta_{n}=2 \pi \Delta \beta n+\Delta
$$

we see that

$$
\hat{H} \psi_{n}=\varepsilon_{\nu}\left(k, \Delta_{n}\right) \psi_{n}+\mathrm{O}(\eta)
$$

where the $\psi_{n}$ are the amplitudes of the generalised Bloch state $|k, \Delta ; \nu\rangle$ and $\varepsilon_{\nu}(k, \Delta)$ is the Bloch dispersion relation for the rational case. Now write

$$
\varepsilon_{\nu}(k, \Delta)=\sum_{m} \varepsilon_{m, \nu}(k) \mathrm{e}^{\mathrm{i} m \Delta q}
$$

so that

$$
\begin{aligned}
\hat{H} \psi_{n} & =\sum_{m} \varepsilon_{m, \nu}(k) \mathrm{e}^{2 \pi i \Delta \beta m n q} \psi_{n}+\mathrm{O}(\eta) \\
& =\sum_{m} \varepsilon_{m, \nu}(k) \mathrm{e}^{\mathrm{i} \kappa m n} \psi_{n}+\mathrm{O}(\eta)
\end{aligned}
$$

(since $\kappa=2 \pi(q \beta-p)=2 \pi q \Delta \beta)$. This implies that

$$
H_{n, \nu}(k)=\varepsilon_{n, \nu}\left(\frac{2 \pi k}{q K_{\nu}}\right)+\mathrm{O}(\eta)
$$

i.e. the terms in (5.7) corresponding to shifting $k$ by $n \kappa$ are equal to the $n$th Fourier coefficient of $\varepsilon_{\nu}(k, \Delta)$ as a function of $\Delta$. 
The coefficients $H_{n, \nu}(k)$ and $N_{n, \nu}(k)$ decay as $|n| \rightarrow \infty$. If the gaps between bands close to within a small quantity $\Delta E$ as $\Delta$ is varied, then $\varepsilon_{\nu}(k, \Delta)$ changes rapidly in the small range $\Delta^{*}=\Delta E$, and the number of Fourier coefficients which are significant is

$$
n_{l}=\mathrm{O}\left(1 / q \Delta^{*}\right)=\mathrm{O}(1 / q \Delta E) .
$$

If the gap $\Delta E$ is not small, then the Fourier coefficients decay over a short range,

$$
n_{l}=\mathrm{O}(1) \text {. }
$$

The generalised Bloch states are a complete set only if the normalisation operator is not singular. The normalisation operator cannot be singular if the product of the number of diagonal elements and their magnitude is small, i.e. if

$$
\eta n_{p} \ll 1 .
$$

If this condition is satisfied, the normalisation operator $\hat{N}$ can be eliminated from the Schrödinger equation by multiplying each side by $\hat{N}^{-1 / 2}$. The resulting Hamiltonian

$$
\hat{H}^{\prime}=\hat{N}^{-1 / 2} \hat{H} \hat{N}^{-1 / 2}
$$

is also a difference operator with periodic coefficients.

To summarise these results: taking matrix elements of the Hamiltonian in a basis of generalised Bloch waves leads to a new Schrödinger equation for the $\nu$ th band, which is also in the form of a difference equation with periodic coefficients. The new ratio of periodicities, $\beta_{\nu}^{\prime}$, is given by (5.9). The matrix element $H_{n, \nu}(k)$ for 'hopping' by $n$ steps is given by the $n$th Fourier coefficient of $\varepsilon_{\nu}(k, \Delta)$ as a function of $\Delta$, plus correction terms (equations (5.16) and (5.14)). The condition for the generalised Bloch waves to form a complete set is (5.19).

The difference equation representing the $\nu$ th band can be obtained by quantising a Hamiltonian $H_{\nu}^{\prime}(x, p)$ given by

$$
H_{\nu}^{\prime}(x, p)=\sum_{n m} H_{n, m}^{(\nu)} \mathrm{e}^{\mathrm{i}(n p+m x)}
$$

where $H_{n, m}^{(\nu)}$ is the $m$ th Fourier coefficient of $H_{n, \nu}(k)$

$$
H_{n, \nu}(k)=\sum_{m} H_{n, m}^{(\nu)} \mathrm{e}^{2 \pi \mathrm{i} m k / K_{\nu}} .
$$

Quantising (5.21) in the $x$ representation using the Weyl quantisation rule gives the difference equation

$$
\sum_{n}\left(\sum_{m} H_{n, m}^{(\nu)} \mathrm{e}^{\mathrm{i} m\left(x+n \hbar_{\nu}^{\prime} / 2\right)}\right) \psi\left(x+n \hbar_{\nu}^{\prime}\right)=E \psi(x)
$$

which is equivalent to $(5.7),(5.8)$ and $(5.20)$ provided

$$
[\hat{x}, \hat{p}]=\mathrm{i} \hbar_{\nu}^{\prime}=2 \pi \mathrm{i} \beta_{\nu}^{\prime} .
$$

Taking matrix elements in a basis of generalised Bloch states therefore leads to a new effective Hamiltonian and Planck constant describing the band. Combining (5.16), (5.21) and (5.22), we find

$$
H_{\nu}^{\prime}(\hat{x}, \hat{p})=\varepsilon_{\nu}(\hat{x} / q, \hat{p} / q)+\mathrm{O}(\eta)
$$

where $\varepsilon_{\nu}(k, \Delta)$ is the Bloch dispersion relation of the band. Equations (5.9), (5.27) and (5.28) are the principal results of this paper. 


\section{Iteration of the RG transformations}

This section will consider the iteration of the renormalisation group transformation introduced in $\$ 5$. The construction of the effective Hamiltonian for the $\nu$ th band depends on the assumptions that the projection operator acts as a small perturbation of the generalised Bloch states, and that the normalisation operator is not singular. Both of these conditions are satisfied if $\Delta \beta$ is sufficiently small, but probably cannot be satisfied in every case. The estimates of how large $\Delta \beta$ can be are discussed below for three different cases: first, where the bands are narrower than the gaps between them, second when the gaps $\Delta E_{\Delta}$ between bands obtained by varying $\Delta$ are narrow, and third when the gaps $\Delta E_{k}$ obtained by varying $k$ are narrow.

(a) Wide gaps. The condition that the projection operator act as a small perturbation of the generalised Bloch states is

$$
\varepsilon=l_{p} / l_{c} \ll 1
$$

where $l_{p}$ is the localisation length of the projection operator and $l_{c}$ the coherence length. These length scales are given by (4.5) and (4.10)

$$
l_{p}=\mathrm{O}(p) \quad l_{c}=\mathrm{O}\left(\frac{\beta}{q \Delta \beta}\right)
$$

so that

$$
\varepsilon=\mathrm{O}\left(q^{2} \Delta \beta\right) \ll 1
$$

The condition for the normalisation operator to be non-singular is given by (5.19)

$$
\varepsilon^{\prime}=n_{i} \eta \ll 1
$$

Using (5.18) and (6.2) this becomes

$$
\varepsilon^{\prime}=\mathrm{O}\left(q^{2} \Delta \beta\right) \ll 1
$$

which is the same as the condition for the projection operator.

(b) $\Delta E_{\Delta}$ is small. When $\Delta E_{\Delta}$ is small, the condition for the projection operator to be a small perturbation is given by (4.5) and (4.12),

$$
l_{p}=\mathrm{O}(p) \quad l_{c}=\mathrm{O}\left(\beta \Delta E_{\Delta} / \Delta \beta\right)
$$

so that

$$
\varepsilon=\mathrm{O}\left(q \Delta \beta / \Delta E_{\Delta}\right) \ll 1
$$

The condition on the normalisation operation again has the same form as for the projection generator.

(c) $\Delta E_{k}$ is small. In this case the localisation length is long instead of the coherence length being short: from (4.6) and (4.10)

$$
l_{p}=\mathrm{O}\left(\beta / \Delta E_{k}\right) \quad l_{c}=\mathrm{O}\left(\frac{\beta}{q \Delta \beta}\right) .
$$


The expression for $\varepsilon$ is of the same form as (6.7):

$$
\varepsilon=\mathrm{O}\left(q \Delta \beta / \Delta E_{k}\right) \ll 1
$$

Again, the condition on $\Delta \beta$ from the requirement that the normalisation operator be non-singular has the same form as for the projection operator.

The condition on $\Delta \beta$ is much more restrictive in cases $(b)$ and $(c)$ than in case (a): when the gaps are wide, the condition (6.3) is not a serious restriction on the applicability of the theory, but when $\Delta E$ is small, it may not be possible to find a suitable rational approximant $p / q$ to the irrational number $\beta$. This suggests a connection between the class of numbers $\beta$ for which the RG transformation can be iterated indefinitely, and the measure of the spectrum. Since the conditions on $\Delta \beta$ when the gaps are small, (6.7) and (6.9), are identical, only two cases must be considered.

\subsection{Wide gaps}

For any irrational number

$$
\beta=\left[n_{1}, n_{2}, n_{3}, \ldots\right]=\frac{1}{n_{1}+\frac{1}{n_{2}+\frac{1}{n_{3}+\ldots}}}
$$

the rational approximants $\beta_{k}=\left[n_{1}, n_{2}, \ldots, n_{k}\right]=p_{k} / q_{k}$ satisfy

$$
\varepsilon_{k}=q_{k}^{2}\left|\Delta \beta_{k}\right|=q_{k}^{2}\left|\beta-p_{k} / q_{k}\right| \leqslant C
$$

for some constant $C$ (Khinchin 1964). This expression should be compared with (6.3). For any irrational number, it is therefore possible to find rational approximants such that $\varepsilon_{k}=\mathrm{O}(1)$. For a typical irrational number, many of the continued fraction coefficients $n_{k}$ are very large, and the corresponding value of $\varepsilon_{k}$ is very small, so that (6.3) is satisfied.

Provided the gaps in the spectrum remain large at every iteration of the RG transformation, it should therefore be possible to iterate this transformation indefinitely for almost all irrational numbers $\beta$. This would imply that the measure of the spectrum is zero, since as the RG transformation is iterated, the measure of the energies which have not been shown to lie in a gap decreases geometrically.

Usually, the gaps do not remain large as the RG transformation is iterated, so that the RG transformation cannot be iterated indefinitely. The exceptional case is when the renormalised Hamiltonians $H_{\nu}^{\prime}(x, p)$ have no open phase trajectories in the phase plane apart from a separatrix (Wilkinson 1984). In this case, semiclassical analysis shows that the bands have total measure of size $O\left(\hbar_{\nu}^{\prime}\right)$, so that the gaps are not small.

This situation, where $H_{\nu}^{\prime}(x, p)$ has no open phase trajectories at every iteration of the RG transformation, occurs when $H(x, p)$ has certain symmetries. If $H(x, p)$ has threefold or fourfold symmetry in the phase plane, there cannot be any open phase trajectories, apart from an isolated separatrix. The Bloch dispersion relations $\varepsilon_{\nu}(k, \Delta)$ have the same rotational symmetry in $k, \Delta$ space as $H(x, p)$ in $x, p$ space, provided the Weyl-Wigner quantisation scheme is used to quantise $H(x, p)$. Then the equation (5.25) shows that the renormalised Hamiltonian $H_{\nu}^{\prime}(x, p)$ has the same rotational symmetry in phase space as $H(x, p)$, apart from the correction term proportional to $\Delta \beta$. 
It has been demonstrated in a special case that the correction term in (3.25) also preserves the phase space symmetry of the Hamiltonian (Wilkinson 1986), and it is likely that this is true in general for the following reason. If $\beta$ is a typical irrational number, some of its continued fraction coefficients are arbitrarily large, and there exist rational approximants $p / q$ for which the correction term in (5.25) is arbitrarily small. As the RG transformation is iterated, there occur Hamiltonians $H_{\nu}^{\prime}(x, p)$ which must therefore be arbitrarily close to having the exact phase space symmetry of $H(x, p)$. This suggests that the correction term in (5.25) always has the same symmetry as $\varepsilon_{\nu}(k, \Delta)$.

To summarise these conclusions: if the RG transformation can be iterated indefinitely for typical values of $\beta$, then the measure of the spectrum is zero. This occurs if the Hamiltonian has centres of threefold or fourfold rotational symmetry in the phase plane, because the spectra of these Hamiltonians have wide gaps, and because these symmetries are preserved by the RG transformation. The RG transformation explains the hierarchically clustered structure of the spectrum of these systems.

\subsection{Narrow gaps}

If the spectrum has a finite measure, and if the RG transformation can be iterated indefinitely, then the size of the gaps must decrease as the iteration proceeds: if the gaps occupy a fraction $\varepsilon_{n}$ of the width of the band at the $n$th iteration, then $\varepsilon_{n}$ must decrease faster than $1 / n$ as $n \rightarrow \infty$. When the gaps become small, the estimate for the allowable size of $\Delta \beta$ is given by (6.7) or (6.9), which is much more restrictive than (6.3). It is not expected, therefore, that the RG transformation can be iterated indefinitely for all irrational $\beta$ if the measure of the spectrum is not equal to zero.

In fact, the size of the gaps is expected to decrease much faster than $1 / n$ : for example, consider the case of Harper's equation when $\alpha \ll 1$. The size of a given gap scales as

$$
\Delta E \sim \alpha^{\mu}
$$

where $\mu$ is one of the gap labelling integers. Results of Bellissard and Simon (1982) show that when $\beta=p / q$, the Bloch dispersion functions $\varepsilon_{\nu}(k, \delta)$ are given by the implicit equation

$$
f_{\alpha}(E)=\cos (k q)+\alpha^{q} \cos (\Delta q)
$$

where $f_{\alpha}(E)$ is a $q$ th degree polynomial. This result shows that as the RG transformation is iterated the Hamiltonian maps into a similar form with $\alpha$ replaced by $\alpha^{\prime}=\alpha^{q}$. Expressed as a fraction of the width of this band, the gaps in the spectrum of the renormalised Hamiltonian are of size

$$
\Delta E \sim q \alpha^{\mu q} \sim q C^{-q}
$$

where $C>1$. Combining (6.14) with (6.7) or (6.9) shows that the RG transformation can only be iterated indefinitely if the rational approximants $p / q$ of $\beta$ all satisfy

$$
\Delta \beta=|\beta-p / q| \leqslant C^{\prime} C^{-q}
$$

where $C^{\prime}, C$ are constants. Irrational numbers satisfying a condition of this type are termed Liouville numbers. It has been shown that when $\beta$ is a Liouville number, the spectrum is of the singular continuous type (Simon 1982), whereas when $\alpha \neq 1$ and $\beta$ is a typical irrational number the eigenfunctions of Harper's equation are either all localised or all extended (for $\alpha>1$ and $\alpha<1$ respectively). The RG transformation described in this paper should also enable the nature of the eigenfunctions in the singular continuous spectrum to be understood. 


\section{Conclusion and discussion}

Bloch electrons in a magnetic field can be modelled by a one-dimensional effective Hamiltonian $H(\hat{x}, \hat{p})$, periodic in $x$ and $p$. The ratio $\beta$ of the area of a flux quantum to the unit cell is equal to $2 \pi \hbar$ divided by the area of the unit cell in phase space. When $\beta$ is rational, $\beta=p / q$, Floquet theory shows that there is a band spectrum with $q$ bands. The energy depends on the Bloch wavevector $k$ and a phase parameter $\Delta$ : $E=\varepsilon_{\nu}(k, \Delta)$.

Generalised Bloch states have been defined for the case in which $\beta$ is perturbed away from the rational case: $\beta=p / q+\Delta \beta$. Provided $\Delta \beta$ is sufficiently small, these states form a complete basis for a given band of the spectrum. The construction of these states depends on the value of the quantised Hall conductance integer, $M_{\nu}$.

Taking matrix elements of the Hamiltonian as a basis of generalised Bloch states leads to a new effective Hamiltonian, $H_{v}^{\prime}(\hat{x}, \hat{p})$, describing the $\nu$ th band, and a new value of $\beta, \beta_{\nu}^{\prime}$. In the limit $\Delta \beta \rightarrow 0, H_{\nu}^{\prime}(\hat{x}, \hat{p})$ is obtained from the Bloch dispersion relation $\varepsilon_{\nu}(k, \Delta)$ by substituting $k \rightarrow \hat{x} / q, \Delta \rightarrow \hat{p} / q$. The transformation $\beta \rightarrow \beta_{\nu}$, given by equation (5.9), depends on the value of the Hall conductance integer $M_{\nu}$. Although the analytical results have only been calculated to lowest order in $\Delta \beta$, the RG transformation could be computed numerically to arbitrary precision, because the gaps in the spectrum ensure that the projection operator is well defined.

The condition on $\Delta \beta$ is that the ratio of the localisation length $l_{p}$ to the coherence length $l_{\mathrm{c}}$ is small (see $\S 4$ ). If the measure of the spectrum is zero, it is possible to find a series of rational approximants such that this RG transformation can be iterated indefinitely, implying that the eigenfunctions have a hierarchical structure, with no characteristic length scale. This occurs if the Hamiltonian has centres of threefold or fourfold symmetry in the phase plane (Claro and Wannier 1979, Wilkinson 1984), but for most other cases the spectrum has finite measure, and the eigenfunctions are either localised, or are simple quasiperiodic functions.

The other situation in which the RG transformation can be iterated indefinitely is when the spectrum does not have measure zero, but when $\beta$ is a Liouville number. In this case the size of the gaps $\Delta E$ in the spectrum decrease as the RG transformation is iterated, and this implies that $\Delta \beta q^{2}$ must get smaller at every iteration.

The results of this paper explain the surprising observations of Hofstadter (1976) about the spectrum of Harper's equation (1.3) when $\alpha=1$. The corresponding Hamiltonian (1.1) has fourfold symmetry in phase space when $\alpha=1$, so that the spectrum is expected to have zero measure for all irrational values of $\beta$. Hofstadter describes the hierarchical structure of the spectrum by an empirical set of rules: if $1 /(q+1)<\beta<$ $1 / q$, the spectrum can be divided into $q$ bands if $q$ is even, $q+1$ bands if $q$ is odd, and the splitting of each band is described by a new value of $\beta$. The new value of $\beta$ is different for the central band. Given that the quantised Hall conductance integer $M_{\nu}$ is unity for the central band and zero for all the rest, it can be seen that Hofstadter's rules are in agreement with equations (5.9) (with $p=1$ ).

The strange properties of the spectrum and eigenfunctions which occur when $H(x, p)$ has centres of threefold or fourfold symmetry in the phase plane are not just a mathematical curiosity, since $H(x, p)$ has the same rotational symmetry as the potential $V(x, y)$, and these are natural crystallographic symmetries in coordinate space (Wilkinson 1987).

None of the earlier proposed RG methods applicable to Bloch electrons in a magnetic field are capable of giving a full description of the spectrum. Various methods have 
been proposed which use localised states as a basis for a band, see e.g. Suslov (1982), Wilkinson (1984). These methods can be developed into an exact RG transformation if the Hall conductance, $M_{\nu}$ is zero, but if $M_{\nu} \neq 0$ it is not possible to use a localised basis set (Wilkinson 1986). Another approach is based on the use of transfer matrices: a renormalisation group transformation is set up to calculate successively longer strings of transfer matrices (Ostlund and Pandit 1984). Using the transfer matrix method it is easy to write down an exact RG transformation when $\beta$ is a quadratic irrational number, but the results only apply to one energy, and give no information about the spectrum as a whole.

An unusual feature of the results described in this paper is that fixed points of the RG transformation are not of very great interest. The 'critical' behaviour of the spectrum observed when $H(x, p)$ has threefold or fourfold symmetry is due to the fact that the RG transformation preserves the symmetry of $H(x, p)$, and is not related to any single unstable fixed point. When the Hamiltonian does not have this critical symmetry, the eigenstates are usually either localised or quasiperiodic, and it might be expected that this corresponds to attractive fixed points of the RG transformation. In these cases the RG transformation breaks down unless the number $\beta$ is a Liouville number, because the gaps in the spectrum become smaller with every iteration.

\section{Acknowledgment}

The award of a research grant by the Nuffield Foundation is gratefully acknowledged. This work was not supported by any military agency.

\section{References}

Bellissard J and Simon B 1982 J. Funct. Anal. 48 408-19

Claro F and Wannier G H 1979 Phys. Rev. B $196068-74$

Harper P G 1955 Proc. Phys. Soc. A 68 879-92

Hofstadter D R 1976 Phys. Rev. B $142239-49$

Johnson R and Moser J 1982 Commun. Math. Phys. 84403

Khinchin A Ya 1964 Continued Fractions (Chicago: University of Chicago Press)

Ostlund S and Pandit R 1984 Phys. Rev. B 29 1394-414

Peierls R 1933 Z. Phys. $80763-91$

Rauh A 1974 Phys. Status Solidi b $65131-5$

1975 Phys. Status Solidi b $699-13$

Simon B 1982 Adv. Appl. Math. 3 463-90

Strěda P 1982 J. Phys. C: Solid State Phys. 15 L717-27

Suslov I M 1982 Zh. Eksp. Teor. Fiz. 83 1079-88 (Sov. Phys.-JETP 56 612-7)

Thouless D J 1983 Phys. Rev. B 27 6083-7

Thouless D J, Kohmoto M, Nightingale M P and den Nijs M 1982 Phys. Rev. Lett. 49 405-8

Wilkinson M 1984 Proc. R. Soc. A $391305-50$

_ 1986 Proc. R. Soc. A 403 135-66

1987 J. Phys. A: Math. Gen. 20 1761-71 